\title{
Granulomatosis with polyangiitis - therapeutic challenge
}

\section{Dorota Szydlarska ${ }^{1}$, Małgorzata Wisłowska}

${ }^{1}$ Family Medicine Outpatient Clinic with Vaccination Centre, Central Clinical Hospital of the Ministry of the Interior, Warsaw, Poland 2Department of Internal Diseases and Rheumatology, Central Clinical Hospital of the Ministry of the Interior, Warsaw, Poland

\begin{abstract}
The antineutrophil cytoplasmic antibody (ANCA) associated vasculitides (AAV) are a group of primary vasculitides that affect predominantly small- to medium-sized blood vessels. The pathology of granulomatosis with polyangiitis (GPA) typically features a granulomatous and sometimes necrotizing vasculitis targeting predominantly the respiratory tract and kidneys, including necrotizing granulomatous inflammation. The diagnosis can be delayed due to the nonspecific nature of the symptoms. Cyclophosphamide and glucocorticoids have been the cornerstone of remission-induction therapy for severe antineutrophil cytoplasmic antibody-associated vasculitis for years. Recently, the monoclonal antibody rituximab was approved for the treatment of GPA, providing an alternative to cyclophosphamide for induction therapy of AAV. In the present case study we used azathioprine as the maintenance drug. However, because of relapse we tried mycophenolate mofetil (MMF) as a second-line drug successfully. This case confirms that MMF is not only well tolerated but also effective in GPA to maintain remission.
\end{abstract}

Key words: mycophenolate mofetil, azathioprine, polyangiitis.

\section{Introduction}

The antineutrophil cytoplasmic antibody (ANCA) associated vasculitides (AAV) are a group of primary vasculitides that affect predominantly small- to medium-sized blood vessels. Granulomatosis with polyangiitis (GPA), formerly Wegener granulomatosis, typically features a granulomatous and sometimes necrotizing vasculitis targeting predominantly the respiratory tract and kidneys including necrotizing granulomatous inflammation [1, 2]. Granulomatosis with polyangiitis is a rare multisystem autoimmune disease of unknown etiology [3]. Granulomatosis with polyangiitis has a spectrum of clinical presentations that includes recurrent respiratory infection, renal and/or ocular manifestations, and nonspecific systemic symptoms. The clinical picture of this disease is not always typical, and due to various symptomatology its diagnosis may be a serious problem, and therefore a wide differential diagnosis is necessary. It should always be remembered that many diseases may imitate GPA. Therefore diagnosis, especially in cases with an atypical clinical course (negative serology, atypical location of organ lesions), has to be carefully evaluated and verified if possible [4]. The diagnostic criteria for granulomatosis with polyangiitis were established by the American College of Rheumatology [5].

Symptoms are often non-specific and diagnosis can be delayed. Limited disease refers to patients with AAV and one or more involved organ systems, but no renal disease or immediately life-threatening complications (Table I). Cyclophosphamide (CYC) and glucocorticoids (GC) have been the cornerstone of remission-induction therapy for antineutrophil cytoplasmic antibody-associated vasculitis for years. Recently, the monoclonal antibody rituximab (RTX) was approved for the treatment of GPA, providing an alternative to CYC for induction therapy of AAV.

\section{Case report}

A 59-year-old woman with a history of hiatal hernia was referred to a general practitioner's office because of

Address for correspondence:

Dorota Szydlarska, Family Medicine Outpatient Clinic with Vaccination Centre, Central Clinical Hospital of the Ministry of the Interior, Wołoska 137, 02-507 Warsaw, Poland, e-mail: dszydlarska@op.pl

Submitted: 13.11.2015; Accepted: 19.01.2016 
Table I. EUVAS disease categorization of ANCA-associated vasculitis [4]

\begin{tabular}{|ll|}
\hline Category & \multicolumn{1}{c|}{ Definition } \\
\hline Localized & $\begin{array}{l}\text { Upper and/or lower respiratory tract disease without any other systemic involvement or constitutional } \\
\text { symptoms }\end{array}$ \\
\hline Early systemic & Any, without organ-threatening or life-threatening disease \\
\hline Generalized & Renal or other organ threatening disease, serum creatinine $<500 \mu \mathrm{mol} / \mathrm{l}(5.6 \mathrm{mg} / \mathrm{dl})$ \\
\hline Severe & Renal or other vital organ failure, serum creatinine $>500 \mu \mathrm{mol} / \mathrm{l}(5.6 \mathrm{mg} / \mathrm{dl})$ \\
\hline Refractory & Progressive disease unresponsive to glucocorticoids and cyclophosphamide \\
\hline
\end{tabular}

cough, joint and muscle pain for about 8 weeks accompanied by elevated body temperature up to $38^{\circ} \mathrm{C}$ lasting for two weeks. Physical examination showed no abnormalities. Blood tests revealed mildly elevated leucocyte count with neutrophilia and elevated inflammatory markers, while urinalysis showed hematuria. Hepatitis and HIV infection were excluded. The concentration of rheumatoid factor and activity of creatine kinase were within references ranges. Chest $X$-ray and ECG were normal. Ultrasound scan of the abdominal cavity was performed but no pathology was found. Empirical antibiotic therapy was administered, but no improvement was observed after 7-day treatment as upper respiratory tract infection was suspected. Several courses of antibiotic therapy (e.g. azithromycin, ciprofloxacin) were administered on an out-patient basis to treat the upper respiratory tract inflammatory disease, but no clinical improvement was obtained. Rheumatological and hematological consultations were planned. However, after 3 months of the lack of effects of antibiotic therapies and persistent elevated body temperature the patient was referred to the Internal Medicine and Rheumatology Department (2010).

Laboratory tests demonstrated elevated inflammatory markers, i.e. erythrocyte sedimentation rate (ESR $88 \mathrm{~mm} / \mathrm{h})$, C-reactive protein level (127 mg/l), and white blood cell count $(27.3 \mathrm{k} / \mu \mathrm{l})$, as well as anemia $(\mathrm{Hgb}$ $11.7 \mathrm{~g} / \mathrm{dl}$ ). The creatinine level was $1.3 \mathrm{mg} / \mathrm{dl}$, while glomerular filtration rate (GFR) was $50.8 \mathrm{ml} / \mathrm{min} / 1.73 \mathrm{~m}^{2}$. CANCA antibody level tested with ELISA significantly exceeded the reference values, while PANCA antibodies, antinuclear antibodies and rheumatoid factor were not found. The activity of creatine kinase was in the reference range. The urinalysis showed hematuria and proteinuria, but there were no clinical data on urinary tract infection. A urine culture was sterile. Additional signs and symptoms were manifested during hospital stay: cough, purulent and bloody discharge from the nose, nocturnal joint and muscle pain, arthritis of the small joints in the hands with several hours of morning stiffness. Chest $X$-ray showed multiple nodules in both lungs, some with cavitation. The patient was consulted by a laryngologist and a nephrologist. The laryngologist performed a biopsy which revealed a multisystemic necrotizing, granulomatous inflammatory process affecting small vessels. The urinalysis showed microscopic hematuria associated with proteinuria. The serum creatinine concentration was elevated (creatinine $1.6 \mathrm{mg} / \mathrm{dl}$, GFR $41.3 \mathrm{ml} / \mathrm{min} / 1.73 \mathrm{~m}^{2}$ ). Therefore the renal biopsy was performed and histopathological examination revealed focal segmental glomerulosclerosis (FSGS).

Table II shows the results of imaging and laboratory tests at different stages of treatment. A diagnosis of granulomatosis with vasculitis was made on the basis of clinical, radiological and histopathological findings. The treatment was started with GC and pulses of CYC $15 \mathrm{mg} / \mathrm{kg}$ per dose every 2 weeks at the beginning ( 3 pulses) and next every 3 weeks for a total dose of $16 \mathrm{~g}$. After treatment with CYC the maintenance therapy with azathioprine (AZA) was started. For a period of 5 years remission had been achieved.

The follow-up visits and examinations were continued at the Rheumatology Department Ambulatory Office. The patient was in good condition, laboratory tests were in the reference ranges, and there were no deviations in imaging studies. In April 2015, the patient showed no dyspnea, no fever, no respiratory tract or ENT disorders, and no skin lesions. The patient was in a good general condition, with vesicular breath sounds on auscultation, soreness and discrete edema of ankles, and degenerative changes.

Laboratory tests showed ESR of $17 \mathrm{~mm} / \mathrm{h}$ and a C-reactive protein level of $13.7 \mathrm{mg} / \mathrm{l}$. However, progression of the pulmonary lesion was observed in CT scans of the chest (large lumps in the superior lobe of the left lung that were not seen previously; Fig. 1).

The treatment was continued with mycophenolate mofetil (MMF) at the dose of $2 \times 500 \mathrm{mg} /$ day for 2 weeks, then $2 \times 1 \mathrm{~g} /$ day, prednisone $7.5 \mathrm{mg} /$ day and trimethoprim/sulfamethoxazole (960 mg/day) as infection prophylaxis was administered. 
Table II. The results of imaging and laboratory tests at different stages of treatment

\begin{tabular}{|c|c|c|c|c|}
\hline & Diagnosis & $\begin{array}{c}\text { Remission after CYC } \\
\text { treatment }\end{array}$ & Relapse & $\begin{array}{c}\text { Remission after MMF } \\
\text { treatment }\end{array}$ \\
\hline Creatine (mg/dl) & 1.6 & 1.1 & 1.7 & 1.0 \\
\hline GFR $\left(\mathrm{ml} / \mathrm{min} / 1.73 \mathrm{~m}^{2}\right)$ & 41.3 & 60.1 & 38.8 & 66.1 \\
\hline CANCA & +++ & + & +++ & + \\
\hline ESR $\mathrm{mm} / \mathrm{h}$ & 88 & 14 & 11 & 7 \\
\hline CRP mg/l & 127 & 6.2 & 13.7 & 2.6 \\
\hline WBC $(\mathrm{k} / \mu \mathrm{l})$ & 27.3 & 11.4 & 24.5 & 10.5 \\
\hline $\mathrm{Hb}(\mathrm{g} / \mathrm{dl})$ & 11.7 & 12.8 & 11.3 & 13.1 \\
\hline Hematuria & + & negative & negative & negative \\
\hline Proteinuria & ++ & negative & negative & negative \\
\hline CT scans & positive & negative & positive & negative \\
\hline
\end{tabular}

After four months, the patient reported good general condition, and denied shortness of breath, joint pain, and fever. The treatment was tolerated well. On physical examination, normal breath sounds were present, joint mobility was preserved, and joint swelling was absent. Laboratory tests showed low ESR $7 \mathrm{~mm} /$ hour, CRP 2.6 $\mathrm{mg} / \mathrm{l}$; blood cell counts and renal and liver tests were normal. The chest CT demonstrated regression of lung lesions (Fig. 2). The treatment has been continued. After four months of treatment the patient did not suffer from side effects of MMF.

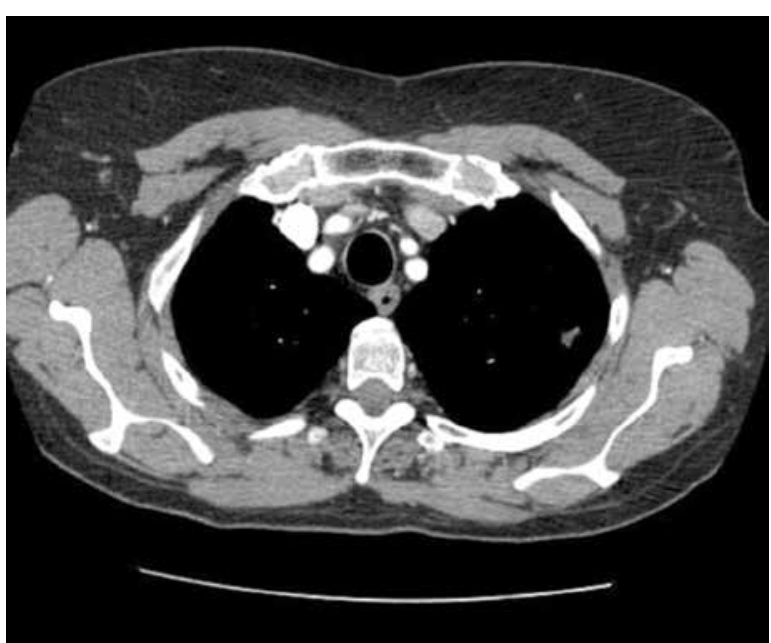

Fig. 1. Areas of consolidation with retraction and band-like extensions towards the pleura are visible in segments $1 / 2$ and 3 of the left lung. These lesions are up to 1.5 and $2 \mathrm{~cm}$ diameter. Multiple minor ill-defined masses are localized in lower lobes of both lungs. Progression is seen in comparison to previous scan.

\section{Discussion}

Granulomatosis with polyangiitis and microscopic polyangiitis are systemic vasculitides with small to medium vessel involvement. Together with idiopathic pauci-immune necrotizing glomerulonephritis, which is considered a renal limited variant of MPA, they are the most common cause of rapidly progressive glomerulonephritis. These entities are frequently associated with ANCA, and are therefore categorized together as AASV [6]. The pathogenesis of granulomatosis with polyangiitis has

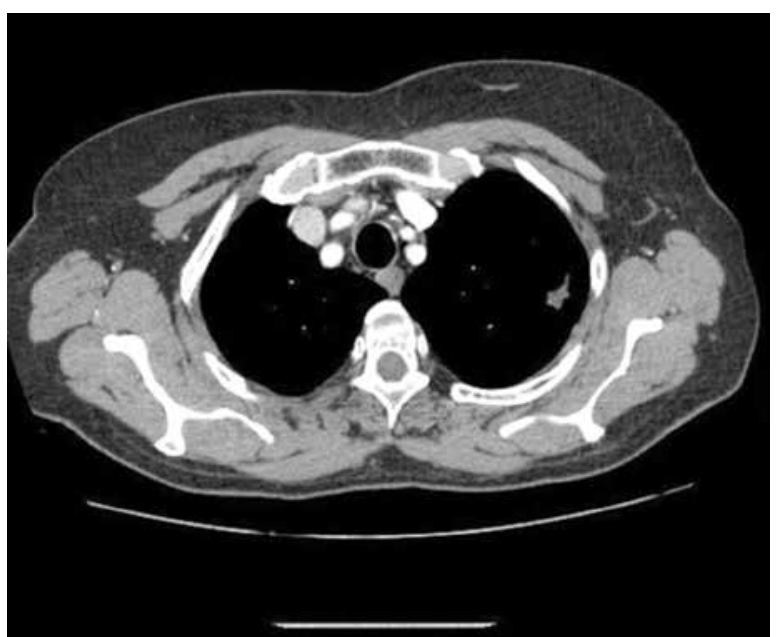

Fig. 2. Regression of nodular masses in segments $1 / 2$ and 3 of the left lung to the size 10 and $12 \mathrm{~mm}$ in diameter. Regression of multiple nodules of both lungs (diameter up to $1 \mathrm{~mm}$ ). Conclusion: partial regression of nodular masses in superior lobe of the left lung, otherwise CT image is comparable to previous examination. 
not been explained completely, but both cellular and humoral components are involved. Circulating ANCA play a key role in the pathogenesis of the disease and may correlate with its activity. GPA is a systemic, necrotizing and granulomatous vasculitis that affects predominantly the upper and lower respiratory tract and the kidney. It is the most common ANCA vasculitis. Fever is a common clinical phenomenon. In most cases, the cause of fever can be determined quickly.

The clinical problem starts when the patient has fever for a long period without any easily detectable cause. The cause of fever can be classified as follows: infectious agent, neoplasm, immune or metabolic disorder, genetic abnormality of inflammasome functioning. The clinical course of fever may vary (continuous, remittent, intermittent, hectic). Detection of the cause of chronic fever is not always possible. Infections, pharyngitis, pneumonia, sarcoidosis, bacterial and infective endocarditis, meningoencephalitis, aseptic meningitis, hepatitis, atypical pneumonia caused by viruses, Chlamydia species, or Mycoplasma tuberculosis, human immunodeficiency virus, abscess, appendicitis, cholecystitis, and carcinoma should be considered in the differential diagnoses. Due to the prevalence of different symptoms, diagnosis is often established quite late. Symptoms of the disease tend to be in varying degrees of severity. At the beginning they may be slight, making the diagnosis of GPA challenges for clinicians.

The diagnosis of GPA is made on clinical manifestations, histological findings and the presence of ANCA in serum. The American College of Rheumatology criteria include: oral and nasal inflammation, abnormal chest radiography (nodules, fixed infiltrates, or cavities), urinary sediment (hematuria), and granulomatous inflammation on biopsy [5]. Treatment of GPA is divided into 2 phases: induction of remission (IR), followed by a maintenance phase (MP). In the IR it is necessary to use intensive immunosuppressive therapy (for example CYC and steroids). The MP is a less intensive therapy in which immunosuppressors (IS) such as AZA, methotrexate (MTX), and MMF, among others, may be employed. Their purpose is to maintain remission and lower the adverse effects associated with IS.

Maintenance therapy is mandatory to reduce the relapse rate. Nowadays, in the majority of patients disease can be brought into remission with CYC and GC. However, depending on disease characteristics patients with AAV have a 29-60\% risk of experiencing relapses of disease within 5 years despite maintenance therapy after induction of remission with less toxic agents, such as AZA, MTX or MMF [7]. More recently, RTX has been found effective in both induction and maintenance of remission in AAV [8]. Azathioprine is the main maintenance drug, although MTX, MMF or leflunomide (LEF) may be used as a second-line drug [9]. Hiemstra et al. [10] showed that relapses were more common in the MMF group (42/76 patients) compared with the AZA group (30/80 patients). In the present case study we tried AZA as the maintenance drug. However, because of relapse we used MMF as a second-line drug successfully.

Cyclophosphamide at the induction phase followed by $A Z A$ is a frequently used therapeutic regimen and is associated with a high remission rate. This regimen is widely used in many centers [11, 12]. Intravenous intermittent administration of CYC is equally effective in inducing remission as daily oral administration and is associated with fewer adverse effects, but the relapse rate is slightly higher (CYCLOPS study) [13]. Cyclophosphamide and GC have been the standard therapy for remission induction for decades [14]. However, not all patients have a remission with this combination of drugs, and those who do often have disease flares requiring repeated treatment. Moreover, patients also suffer from side effects of CYC, including infertility, cytopenia, infections, bladder injury, and cancer, as well as the multiple adverse effects of lengthy courses of glucocorticoid treatment. For remission maintenance the choice may be AZA or MMF.

Azathioprine is an immunosuppressive drug used in organ transplantation and autoimmune disease and belongs to the chemical class of purine analogues [15]. The main adverse effect of AZA is bone marrow suppression, which can be life-threatening. Azathioprine is also listed by the International Agency for Research on Cancer as a group 1 carcinogen (carcinogenic to humans). Methotrexate is equivalent to AZA for maintenance therapy, but adverse effects are not lower [16]. Its prescription should be restricted to patients with a localized form of GPA and during the maintenance period $[12,17]$.

In generalized, non-organ-threatening disease, remission can be induced with methotrexate and steroids, where the steroid dose is reduced after a remission has been achieved and MTX used as maintenance. In the case of organ-threatening disease, intravenous CYC with $\mathrm{GC}$ is recommended. Once remission has been achieved, AZA and GC can be used to maintain remission. In severe kidney vasculitis, the same regimen is used but also plasma exchange may be needed. In pulmonary hemorrhage, high doses of CYC with intravenous methylprednisolone may be used, or alternatively CYC, steroids, and plasma exchange. Randomized controlled trials have shown that RTX is non-inferior to CYC followed by AZA (CYC/AZA) for remission induction in severe GPA and MPA [17]. Current approaches to treatment in induction and maintenance of AAV are well established (Table III) [18]. 
Table III. Suggested schema for the management of ANCA-associated vasculitis, adapted and updated from Pallan et al. [22]

\begin{tabular}{|c|c|c|c|}
\hline Disease severity & EUVAS definition & Induction therapy & Maintenance therapy \\
\hline Localized & $\begin{array}{l}\text { Upper and/or lower respiratory tract } \\
\text { disease without any other systemic in- } \\
\text { volvement or constitutional symptoms }\end{array}$ & MTX and GKS & $\begin{array}{l}\text { Low-dose steroids plus AZA or } \\
\text { LEF or MTX (+ trimethoprim-sul- } \\
\text { phamethoxazole also added) }\end{array}$ \\
\hline Early systemic & $\begin{array}{l}\text { Any, without organ-threatening or life- } \\
\text { threatening disease }\end{array}$ & MTX or CYC and GKS & $\begin{array}{l}\text { Low-dose steroids plus AZA or } \\
\text { MTX }\end{array}$ \\
\hline Generalized & $\begin{array}{l}\text { Renal or other organ threatening dis- } \\
\text { ease, serum creatinine }<500 \mu \mathrm{mol} / / \\
(5.6 \mathrm{mg} / \mathrm{dl})\end{array}$ & CYC (or RTX) and GKS & $\begin{array}{l}\text { Low-dose steroids plus AZA } \\
\text { MMF as second-line agent }\end{array}$ \\
\hline Severe & $\begin{array}{l}\text { Renal or other vital organ failure, serum } \\
\text { creatinine }>500 \mu \mathrm{mol} / \mathrm{l}(5.6 \mathrm{mg} / \mathrm{dl})\end{array}$ & $\begin{array}{l}\text { CYC and steroids plus plasma } \\
\text { exchange (RTX instead of CYC) }\end{array}$ & $\begin{array}{l}\text { Low-dose steroids plus AZA } \\
\text { MMF as second-line agent }\end{array}$ \\
\hline Refractory & $\begin{array}{l}\text { Progressive disease unresponsive to } \\
\text { GC and cyclophosphamide }\end{array}$ & $\begin{array}{l}\text { Deoxyspergualin, antithymo- } \\
\text { cyte globulin, or RTX }\end{array}$ & No consensus \\
\hline
\end{tabular}

Mycophenolate mofetil, an immune suppressive initially introduced for the prevention of solid organ allograft rejection, is increasingly used in autoimmune conditions, including vasculitis. Varying efficacy of MMF in ANCA-associated systemic vasculitides was observed, with over $50 \%$ of patients with relapsing disease achieving remission and marked falls in concomitant steroid doses. However, longer follow-up indicated a subsequent relapse rate of over $50 \%$ that may be associated with low MMF dosing [19]. In patients with ANCA-associated systemic vasculitides and renal involvement, MMF seems to be an effective and well-tolerated option in sustaining short- and medium-term remission [20]. The use of MMF in autoimmune disease is often limited by adverse effects. However, all patients should know that the side effects of MMF may be constipation, nausea, headache, diarrhea, vomiting, stomach upset, loss of appetite, bloating or trouble sleeping. Mycophenolate mofetil weakens the immune system and may make patients more likely to develop infections. Mycophenolate mofetil is a strong immunosuppressive drug with rather low toxicity due to its lymphocyte-selective mode of action [21].

Rituximab constitutes promising therapy for refractory GPA [22]. Rituximab associated with glucocorticoid treatment adapted for disease severity appeared to induce remission effectively in GPA patients. Maintenance treatment with low doses of RTX in a routine timebased protocol is safe and associated with low rates of relapse on treatment [23]. Rituximab is an effective remission-inducing agent in GPA. The addition of a conventional maintenance agent to RTX and GCs decreased the incidence of relapse and did not result in a higher incidence of adverse events [24]. Rituximab is generally well tolerated, but side effects include leucopenia and infection.
The European League against Rheumatism (EULAR) guidelines for the management of small and medium vessel vasculitis have been published [25]. The success and safety profile of RTX in refractory disease has led to trials in maintenance and induction therapy which may see it recommended as standard practice, although high cost may limit its use. The wide range of newer biologic agents now available brings huge possibilities for immunotherapy in relapsing or refractory disease. The RITUXVAS trial reported similar remission induction rates and safety between RTX- and CYC-based regimens for ANCA-associated vasculitis at 12 months [26]. Duration of response to RTX can be variable but is frequently prolonged, with a median time of 12-16 months [27].

The Rituximab in ANCA-Associated Vasculitis (RAVE) trial was a multicenter, randomized, placebo-controlled trial that compared RTX for remission induction and maintenance with standard therapy consisting of CYC followed by AZA in patients with severe AAV [28]. Treatment with RTX plus GCS would not be inferior to daily CYC plus GCs for remission induction and would permit discontinuation of prednisone within 6 months [28]. The RAVE trial showed that RTX matched the efficacy of CYC (standard therapy) in inducing remission in patients with severe AAV. The results held true for subsets of patients with major renal disease and those with alveolar hemorrhage. There were no significant differences in flare rates at 6 months and no difference in the rate of severe adverse events. However, participants receiving CYC experienced more selected adverse events, particularly leukopenia. Due to the high mortality rate in GPA (28.5\% in our patients), aggressive treatment is needed sometimes [29]. Previous randomized trials and clinical observations show some possible limitations of treatment with CYC and GCS, so RTX seems to be a good 
alternative in those patients in induction therapy as well as in maintenance therapy [30,31].

\section{Summary}

In conclusion, this case confirms that MMF is not only well tolerated but is also effective in GPA to maintain remission. Therapy should be chosen individually, depending not only on the stage and severity of the disease, but also on genetic and some prognostic factors.

\section{The authors declare no conflict of interest.}

\section{References}

1. Muller K, Lin JH. Orbital granulomatosis with polyangiitis (Wegener granulomatosis): clinical and pathologic findings. Arch Pathol Lab Med 2014; 138: 1110-1114.

2. Rossini BA, Bogaz EA, Yonamine FK, et al. Refractory otitis media as the first manifestation of Wegener's granulomatosis. Braz J Otorhinolaryngol 2010; 76: 541.

3. European therapeutic trials in ANCA-associated systemic vasculitis: Disease scoring, consensus regimens and proposed clinical trials. European Community Study Group on Clinical Trials in Systemic Vasculitis ECSYSVASTRIAL. Clin Exp Immunol 1995; 101 Suppl 1: 29-34.

4. Madej M, Matuszewska A, Białowąs K, Wiland P. Ziarniniakowatość z zapaleniem naczyń - problemy diagnostyczne? Reumatologia 2014; 52: 344-346.

5. Leavitt RY, Fauci AS, Bloch DA, et al. The American College of Rheumatology 1990 criteria for the classification of Wegener's granulomatosis. Arthritis Rheum 1990; 33: 1101-1107.

6. Jennette JC, Falk RJ, Andrassy K, et al. Nomenclature of systemic vasculitides: Proposal of an international consensus conference. Arthritis Rheum 1994; 39: 189-195.

7. De Joode AA, Anders AA, Rutgers A, et al. Maintenance therapy in antineutrophil cytoplasmic antibody-associated vasculitis: who needs what and for how long? Nephrol Dial Transplant 2015; 30: 150-158.

8. McGregor JG, Hogan SL, Kotzen ES, et al. Rituximab as an immunosuppressant in antineutrophil cytoplasmic antibody-associatedvasculitis. Nephrol Dial Transplant 2015; 30: 123-131.

9. Puéchal X. Antineutrophil cytoplasmic antibody-associated vasculitides. Joint Bone Spine 2007; 74: 427-435.

10. Hiemstra TF, Walsh M, Mahr A, et al. European Vasculitis Study Group (EUVAS). Mycophenolate mofetil vs azathioprine for remission maintenance in antineutrophil cytoplasmic antibody-associated vasculitis: a randomized controlled trial. JAMA 2010; 304: 2381-2388.

11. de Groot K, Harper L, Jayne DR, et al. Pulse versus daily oral cyclophosphamide for induction of remission in antineutrophil cytoplasmic antibody-associated vasculitis: a randomized trial. Ann Intern Med 2009; 150: 670-680.

12. Pagnoux C, Mahr A, Hamidou MA, et al. Azathioprine or methotrexate maintenance for ANCA-associated vasculitis. N Engl J Med 2008; 359: 2790-2803.
13. Guillevin L, Lhote F, Cohen P, et al. A prospective, randomized trial comparing steroids and pulse cyclophosphamide versus steroids and oral cyclophosphamide in the treatment of generalized Wegener's granulomatosis. Arthritis Rheum 1997; 40: 2187-2198.

14. Fauci AS, Wolff SM. Wegener's granulomatosis: studies in eighteen patients and a review of the literature. Medicine (Baltimore) 1973; 52: 535-561.

15. Sharaf PH, Yazici Y. Necrotizing vasculitis - a 2009 update. Bull NYU Hosp Jt Dis 2009; 67: 303-305.

16. Nachman PH. Vasculitis syndromes: which maintenance therapy for ANCA vasculitis? Nat Rev Nephrol 2009; 5: 254-256.

17. Specks U. Pro: Should all patients with anti-neutrophil cytoplasmic antibody-associated vasculitis be primarily treated with rituximab? Nephrol Dial Transplant 2015; 30: 10831087.

18. Pallan L, Savage CO, Harper L. ANCA-associated vasculitis: from bench research to novel treatments. Nat Rev Nephrol 2009; 5: 278-286.

19. Koukoulaki M, Jayne DR. Mycophenolate mofetil in anti-neutrophil cytoplasm antibodies-associated systemic vasculitis. Nephron Clin Pract 2006; 102: 100-107.

20. Iatrou C, Zembala S, Ravela I, et al. Mycophenolate mofetil as maintenance therapy in patients with vasculitis and renal involvement. Clin Nephrol 2009; 72: 31-37.

21. Van der Woude FJ. Mycophenolate mofetil (RS 61443): Nothing new under the sun or an important break-through in the field of transplantation? Nephrol Dial Transplant 1995; 10: 1112-1115.

22. Vera-Lastra O, Olivera-Acevedo A, McDonal-Vera A, et al. Wegener's granulomatosis: diagnosis and treatment. Gac Med Mex 2009; 145: 121-129.

23. Calich AL, Puéchal X, Pugnet G, et al. French Vasculitis Study Group. Rituximab forinduction and maintenance therapy in granulomatosis with polyangiitis (Wegener's). Results of a single-center cohort study on 66 patients. J Autoimmun 2014; 50: 135-141.

24. Azar L, Springer J, Langford CA, et al. Rituximab with or without a conventional maintenance agent in the treatment of relapsing granulomatosis with polyangiitis (Wegener's): a retrospective single-center study. Arthritis Rheum 2014; 66: 2862-2870.

25. Mukhtyar C, Guillevin L, Cid MC, et al. EULAR recommendations for the management of primary small and medium vessel vasculitis. Ann Rheum Dis 2009; 68: 310-317.

26. Jones RB, Furuta S, Tervaert JW, et al. European Vasculitis Society (EUVAS). Rituximab versus cyclophosphamide in ANCA-associated renal vasculitis: 2-year results of a randomised trial. Ann Rheum Dis 2015; 74: 1178-1182.

27. Smith KG, Jones RB, Burns SM, et al. Long-term comparison of rituximab treatment for refractory systemic lupus erythematosus and vasculitis: Remission, relapse, and re-treatment. Arthritis Rheum 2006; 54: 2970-2982.

28. Stone JH, Merkel PA, Spiera R, et al. Rituximab versus cyclophosphamide for ANCA-associated vasculitis. N Engl J Med 2010; 363: 221-232.

29. Wisłowska M, Gozdowska J. Ziarniniak Wegenera - obserwacja przypadków. Reumatologia 2008; 46: 68-71. 
30. Zdrojewski Z. Postępy w patogenezie, diagnostyce i leczeniu układowych zapaleń naczyń związanych z przeciwciałami przeciwgranulocytarnymi. Roczniki Pomorskiej Akademii Medycznej w Szczecinie 2014; 2: 44-49.

31. Zdrojewski Z. Indywidualizacja leczenia zapaleń naczyń związanych z przeciwciałami przeciwgranulocytarnymi. Reumatologia 2014; 52: 221-223. 